\title{
Graded-Gap a-SiC:H p-i-n Thin-Film Light-Emitting Diodes
}

\author{
Jyh-Wong Hong, Nerng-Fu Shin, Tean-Sen Jen, Sui-Liang Ning, and \\ Chun-Yen Chang, Fellow, IEEE
}

\begin{abstract}
In order to improve the performance of hydrogenated amorphous-silicon carbide (a-SiC:H) p-i-n thin-film light-emitting diodes (TFLED's), a p-i-n TFLED with a graded $p-i$ junction has been proposed and fabricated. The electroluminescence (EL) intensity of the proposed TFLED was more than 100 times higher than that of the basic p-i-n TFLED and about 35 times lower than that of the conventional green LED, at the same injection current density. This significant improvement was attributed to the better interface property and enhancement of hole injection efficiency by using the graded-gap p-i junction.
\end{abstract}

\section{INTRODUCTION}

$\mathrm{T}$ HE development of a-SiC:H p-i-n TFLED has made noticeable progress in recent years [1]-[5]. It has several advantages as compared to the crystal LED, such as accessibility to large-area flat-panel displays, tunable color by using integrated multilayer structures, and lower cost. However, to meet the requirements of practical applications, it needs performance improvements, including the increase of electroluminescence (EL) intensity and the enhanced emission of light with shorter wavelength (green to blue). The graded-gap p-i interface was employed in this study to improve its EL intensity.

\section{DeVice FAbrication ANd Operation}

The employed substrate was indium-tin-oxide (ITO) coated Corning 7059 glass. After the standard cleaning process, it was put into the ULVAC CPD-1108D plasmaenhanced chemical vapor deposition (PECVD) system. In order to reduce the contact resistance between the ITO electrode and the p-type a-SiC:H layer, the $\mathrm{CF}_{4}-\mathrm{O}_{2}$ plasma dry-etching pretreatment was used to bombard the ITO film before the deposition of the p-type a-SiC:H layer [4], [6].

The schematic cross section of the proposed a-SiC:H TFLED is shown in Fig. 1(a). Its graded-gap p-i junction was achieved by terminating the $\mathrm{B}_{2} \mathrm{H}_{6}$ dopant during continuous deposition of a-SiC:H films. As shown in the figure, the thickness of the graded region was approximately equal to $30 \AA$ as estimated by using the equilib-

Manuscript received February 3, 1992. This work was supported by the National Science Council, Republic of China.

J.-W. Hong, N.-F. Shin, T.-S. Jen, and S.-L. Ning are with the Department of Electrical Engineering, National Central University, Chung-Li, Taiwan, 320, Republic of China.

C.-Y. Chang is with the Institute of Electronics, National Chiao-Tung University, Hsin-chu, Taiwan, 300, Republic of China.

IEEE Log Number 9201779.

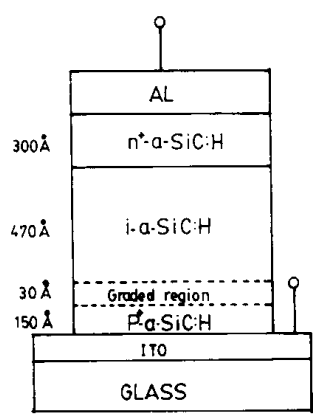

(a)

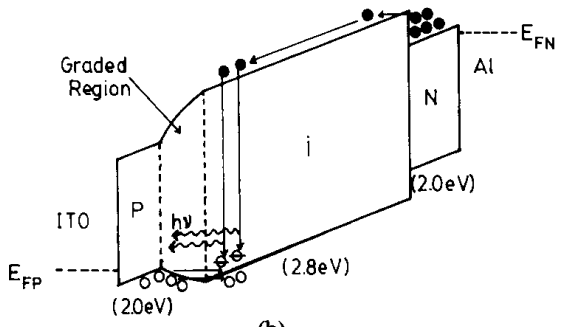

(b)

Fig. 1. (a) Schematic cross section of the graded-gap a-SiC:H p-i-n TFLED and (b) its schematic energy band diagram under forward bias.

rium mass-transport continuity equation [7]. The deposition conditions for various layers of the graded-gap aSiC:H TFLED are summarized in Table I. Finally, the $5000-\AA \mathrm{Al}$ top electrode with a circular area of $1.41 \times$ $10^{-2} \mathrm{~cm}^{2}$ was deposited by employing thermal evaporation through a metal mask.

Fig. 1(b) shows the schematic energy-band diagram under forward bias for this fabricated TFLED. The optical gap of the doped layers is limited to $2.0 \mathrm{eV}$ due to the valence-electron controllability [8], and the optical gap of the employed intrinsic layer is $2.8 \mathrm{eV}$ [7]. So, the proposed graded-gap p-i junction could be used to minimize the effect of notch barrier height on hole transport, since the current transport of the $\mathrm{p}-\mathrm{i}-\mathrm{n}$ a-SiC:H TFLED is determined by the carriers tunneling through triangular barriers at the $p-i$ and $i-n$ interfaces and the emission of light is mainly based on the radiative recombination in the i-layer [9]. The trapping centers at the previously used step p-i interface, resulting from the interruption of $R F$ power during the depositions of device's layers, degrade the EL intensity due to the nonradiative recombination. The 
TABLE I

DePosition CONDITIONS FOR VARIOUS LAYERS OF a-SiC: $\mathrm{H}$ GRADED-GAP p-i-n TFLED

\begin{tabular}{|c|c|c|c|c|}
\hline Layer & $\begin{array}{l}\text { Flow-rate ratio } \\
\quad(\mathrm{sccm})\end{array}$ & $\begin{array}{l}\text { Pressure } \\
\text { (torr) }\end{array}$ & $\begin{array}{l}\text { RF power } \\
\text { (W) }\end{array}$ & $\begin{array}{c}\text { Deposition rate } \\
(\AA / \mathrm{s})\end{array}$ \\
\hline $\mathrm{p}$ & $\begin{array}{l}\mathrm{SiH}_{4}^{1}: \mathrm{C}_{2} \mathrm{H}_{2}: \mathrm{B}_{2} \mathrm{H}_{6}^{2} \\
=200: 6: 38\end{array}$ & 0.3 & 20 & 1.6 \\
\hline \multirow{2}{*}{$\begin{array}{l}\mathrm{i} \\
\mathrm{n}\end{array}$} & $\mathrm{SiH}_{4}: \mathrm{C}_{2} \mathrm{H}_{2}=200: 6$ & 0.3 & 20 & 1.25 \\
\hline & $\begin{array}{l}=200: 8: 72 \\
=1 n_{3}\end{array}$ & 1.0 & 35 & 1.4 \\
\hline
\end{tabular}

Substrate temperature: $180^{\circ} \mathrm{C}$

1: $96 \% \mathrm{H}_{2}+4 \% \mathrm{SiH}_{4}$

2: $99 \% \mathrm{H}_{2}+1 \% \mathrm{~B}_{2} \mathrm{H}_{6}$

3: $99 \% \mathrm{H}_{2}+1 \% \mathrm{PH}_{3}$

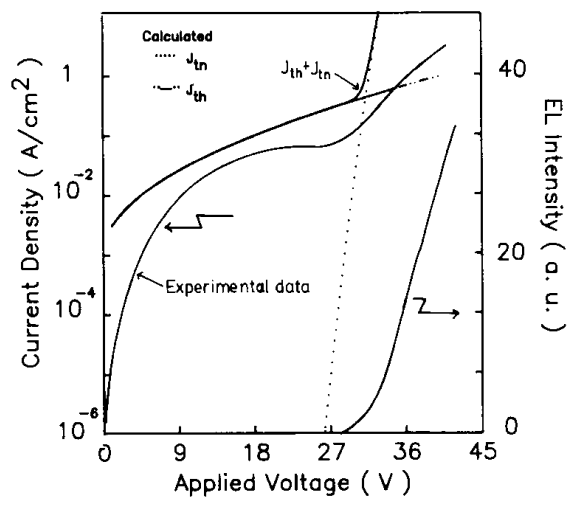

Fig. 2. The relationships of EL intensity and injection current density versus applied voltage for the graded-gap a-SiC: $\mathrm{H}$ p-i-n TFLED (solid lines). The calculated thermionic current $J_{t h}$ under low bias and electron tunneling current $J_{t n}$ under high bias are also indicated (dashed lines).

graded-gap p-i junction could be employed to obtain a better interface property by continuous deposition process using $\mathrm{C}_{2} \mathrm{H}_{2}$ as the carbon source.

\section{EXPERIMENTAL RESULTS}

Fig. 2 shows the relationships of EL intensity and injection current density versus applied voltage for the graded-gap a-SiC:H p-i-n TFLED. As can be seen, the tunneling current occurs at an applied voltage equal to 27 $\mathrm{V}$ where the EL intensity begins to increase. This tunneling phenomenon was checked by the linear $\log \left(J / V^{2}\right)$ versus $(1 / V)$ relationship for $V \geq 27 \mathrm{~V}$ following the Fowler-Nordheim formula [5], [9], [10], where $J$ is the current density and $V$ is the applied voltage. The calculated results (dashed-line) are also shown in this figure. The injection current is dominated by the thermionic emission current $J_{t h}$ under low bias, but the electron tunneling current $J_{t n}$ dominates the current flow when the applied voltage exceeds $27 \mathrm{~V}$. Because the valenceband discontinuity $\Delta E_{v}(0.56 \mathrm{eV})$ is about 3 times larger than conduction-band discontinuity $\Delta E_{c}(0.18 \mathrm{eV})$ [9], the hole tunneling current is relatively very small under device operation voltage range. However, since the luminescence process is based on the radiative recombination of

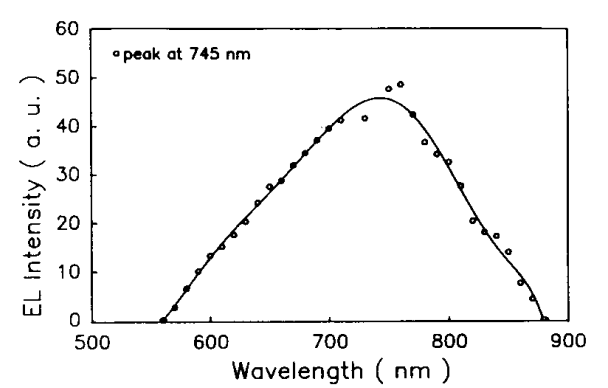

Fig. 3. EL spectrum of graded-gap a-SiC:H p-i-n TFLED.

electrons and holes injected into the i-layer, the hole injection efficiency should determine the EL intensity [4]. Therefore, enhancement of the hole tunneling current will obtain a higher EL intensity. In the graded-gap p-i structure, the effective barrier height at the $p$-i junction is lower than that of the abrupt $\mathrm{p}$-i junction. So the injection efficiency of the hole can be improved, and hence it results in an increase of EL intensity.

Fig. 3 shows the EL spectrum of the graded-gap a-SiC:H p-i-n TFLED under a pulse voltage bias $(0-30 \mathrm{~V}, 200 \mathrm{~Hz}$, $50 \%$ duty cycle), measured by using a monochrometer (ORIEL 77200), a photomultiplier (ORIEL 7070), and a lock-in amplifier. The peak of the EL spectrum is located at $745 \mathrm{~nm}$, which is in the red-light region. It shows a red-orange color experimentally. It is also evident that about one-half of the EL emission lays in the infrared region and cannot be observed by the naked eye. If the peak of the EL spectrum was moved to a shorter wavelength by increasing the carbon content of the i-layer, the EL spectrum could be blue-shifted further [9]. The abrupt $\mathrm{p}$-i-n TFLED shows a red color (peak is around $750 \mathrm{~nm}$ and FWHM is about $190 \mathrm{~nm}$ ) while the hot-carrier tunneling injection (HTI) TFLED shows an orange one (peak is around $700 \mathrm{~nm}$ and FWHM is about $200 \mathrm{~nm}$ ) [6]. The HTI structure is achieved by inserting the thin $(100 \AA)$ higher gap a-SiC: $\mathrm{H}$ layers at the $\mathrm{p}-\mathrm{i}$ and $\mathrm{i}-\mathrm{n}$ interfaces to improve the luminosity of the TFLED by enhancing the injection efficiencies of electrons and holes [5], [6].

Fig. 4 shows a summary of the EL intensities of the a-SiC:H p-i-n TFLED, HTI TFLED [6], graded-gap p-i-n TFLED, and conventional green LED. The EL intensity of the TFLED was measured by placing the TFLED in front of a photomultiplier (ORIEL 7070) driven by a HP 4145B semiconductor parameter analyzer. As can be seen from the figure, the EL intensity of the graded-gap TFLED is more than 100 times $\left(\sim 28 / 0.28\right.$ at $\left.100 \mathrm{~mA} / \mathrm{cm}^{2}\right)$ higher than that of the abrupt $\mathrm{p}-\mathrm{i}-\mathrm{n}$ TFLED and about 35 times $\left(\sim 1000 / 28\right.$ at $\left.100 \mathrm{~mA} / \mathrm{cm}^{2}\right)$ lower than that of the conventional green LED (with a device area of $2.5 \times 10^{-3}$ $\mathrm{cm}^{2}$ ) under the same injection current density ranging from 100 to $1000 \mathrm{~mA} / \mathrm{cm}^{2}$.

It was also found that the graded-gap TFLED's showed a stable light emission even under a continuous dc bias, but the abrupt-gap one was burned out occasionally. 


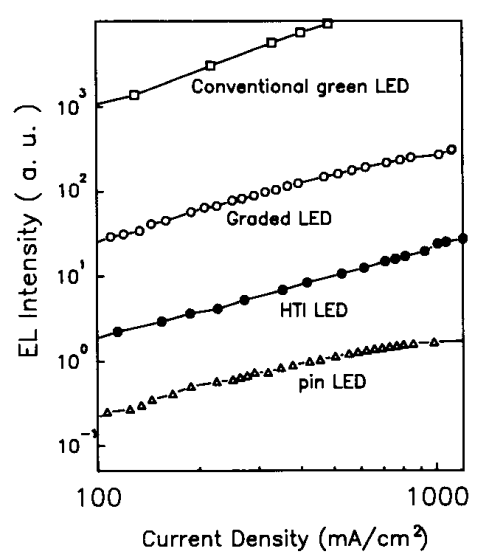

Fig. 4. The $\log -\log$ plot of EL intensities versus injection current density for a-SiC:H p-i-n TFLED, HTI TFLED [6], graded-gap p-i-n TFLED, and conventional green LED.

\section{CONCLUSION}

As an approach to improve the EL intensity of a $\mathrm{SiC}: \mathrm{H}$ $\mathrm{p}$-i-n TFLED, the graded-gap $\mathrm{p}-\mathrm{i}$ junction was used to improve interface properties, hole injection efficiency, and EL intensity substantially. The reliability of a-SiC:H TFLED was improved as EL intensity was increased; this was because the heat-generating nonradiative recombination of carriers deteriorated the reliability of the TFLED. Also, the color of emission light of the proposed TFLED could be modified by the content of carbon in the luminescent a-SiC:H i-layer. By employing various technolo- gies, such as achievable high-performance films by electron cyclotron resonance (ECR) CVD [7] to reduce the nonradiative recombination further, the practical application of a-SiC:H TFLED is foreseeable.

\section{REFERENCES}

[1] H. Matunami and M. Yoshimoto, "Polarization effects in AC electroluminescence of a-SiC:H," J. Non-Cryst. Solids, vol. 59, pp. $569-572,1983$.

[2] H. Munekata and H. Kukimoto, "Electroluminescence in hydrogenated amorphous silicon-carbon alloy," Appl. Phys. Lett., vol. 42, no. 5 , pp. 432-434, 1983.

[3] D. Kruangam, T. Endo, G. P. Wei, H. Okamoto, and Y. Hamakawa, "Amorphous silicon-carbide thin film light emitting diode," Japan. J. Appl. Phys., vol. 24, no 10, p. L806, 1985.

[4] D. Kruangam et al., "Amorphous silicon-carbide thin film light emitting diode," Optoelectron. Decice Technologies, vol. 1, no. 1, pp. 67-84, 1986.

[5] S. M. Paasche, T. Toyama, H. Okamoto, and Y. Hamakawa, "Amorphous-SiC thin film p-i-n light-emitting diode using amorphous-SiN hot-carrier tunneling injection layers," IEEE Trans. Electron Devices, vol. 36, no. 12, pp. 2895-2902, 1989.

[6] J. W. Hong, T. S. Jen, N. F. Shin, J. D. Lee, and C. Y. Chang, "Visible a-SiC:H p-i-n light emitting diodes with hot-carrier tunneling injection layers," to be published in J. Chinese Inst. Eng., Series $A$.

[7] S. L. Ning, "Design and fabrication of amorphous graded-gap and quantum-well-injection p-i-n TFLED's," M.S. thesis, National Central Univ., Taiwan, R. O. C., 1991.

[8] Y. Hamakawa et al.. "Toward a visible light display by amorphous $\mathrm{SiC}: \mathrm{H}$ alloy system," Optoelectron. Derices Technologies, vol. 4, no 2, pp. 281-294, 1989.

[9] D. Kruangam, M. Deguchi, T. Toyama, H. Okamoto, and Y. Hamakawa, "Carrier injection mechanism in an a-SiC p-i-n junction thin film LED," IEEE Trans. Electron Devices, vol. 35, no. 7, pp. 957-964, 1988.

[10] S. M. Sze, Physics of Semiconductor Devices, 2nd ed. New York: Wiley, 1981, p. 403. 